\begin{tabular}{|c|c|c|}
\hline Beitr. Ent. & Keltern & ISSN 0005-805X \\
\hline $\mathbf{5 6}(2006) 2$ & S. $387-403$ & 15.12 .2006 \\
\hline
\end{tabular}

\title{
PhyloCode und DNA Barcoding - Taxonomische Regeln und Techniken im Wandel?
}

\author{
Mit 11 Figuren
}

Peter Nagel

\section{Zusammenfassung}

Die Begriffe PhyloCode und DNA Barcoding stehen für innerhalb der Wissenschaftsgemeinschaft heftig diskutierte, jüngere Entwicklungen, die insbesondere für die Taxonomie (Systematik) und unmittelbar darauf aufbauende Disziplinen wie die Biogeographie grosse Relevanz haben.

Der PhyloCode wurde als internationales Regelwerk für die phylogenetische Nomenklatur gegründet. Seine Ziele beinhalten die Einführung eines universell für alle Organismen gültigen Systems auf der Basis der phylogenetischen Beziehungen. Existierende Normen wie die Internationalen Regeln für die zoologische Nomenklatur (ICZN) sind primär merkmals- und rang-basiert und ermöglichen kein detailliertes Abbild der phylogenetischen Beziehungen. Der PhyloCode nennt Regeln für die Benennung von Monophyla und anderen Kladen, verzichtet aber auf jede Information über die relative hierarchische Position der Abstammungsgemeinschaften. Die angeblich im Gegensatz zu den existierenden Regelwerken vorhandene Stabilität der Nomenklatur kann nicht nachvollzogen werden, da sich die als Begründungen verwendeten Synapomorphien bei Revisionen als Homoplasien herausstellen können. Auch die Prüfung weiterer Punkte des PhyloCode ergab keine Hinweise auf belegbare Vorteile im Vergleich zu einem System wie dem ICZN, das durchaus Defizite in Bezug auf die Erkenntnisfortschritte der phylogenetischen Forschung aufzuweisen hat, das sich aber im Prinzip seit 250 Jahren und als offizielles Regelwerk seit 100 Jahren bewährt hat.

Das DNA Barcoding („Strichcode des Lebens"), steht für eine Genabschnitt-Methode zur Kennzeichnung aller Arten. Das mitochondriale Gen COI wurde als der DNA-Abschnitt bestimmt, mit dessen Hilfe sich theoretisch jeder Organismus eindeutig beschreiben und identifizieren lassen soll. Die Analyse und Datenbankspeicherung der COI-Sequenz aller Arten wurde als effektivere Methode zur Beschreibung der taxonomischen Biodiversität bezeichnet als die derzeit verwendeten, oft nicht-molekularen Methoden der traditonellen Taxonomen. Allerdings ist die COI-bezogene molekulare Analyse sämtlicher bekannter Arten utopisch. Weiterhin können sich die Basensequenzen der Proben von zwei identischen Taxa so sehr unterscheiden, dass eine sichere Bestimmung nicht immer möglich ist. Die für einige Taxa bereits intensiv betriebene COI-Analyse kommt auch der integrativen Taxonomie zugute, die bereits seit längerem schon molekulargenetische neben den traditionellen Methoden verwendet. Das Ziel des DNA Barcoding, nämlich das automatische Identifizieren von Taxa, ist nur in manchen Fällen eindeutig möglich und kann die integrativ arbeitenden Taxonomen nicht ersetzen.

\section{Summary}

The terms PhyloCode and DNA Barcoding represent novel, strongly debated developments of high significance to taxonomy (systematics) and directly linked disciplines like biogeography.

The PhyloCode has been set up as an international code of phylogenetic nomenclature. Its goals comprise the introduction of a taxonomic system which builds on the phylogenetic relationships and which is universally applicable to all organisms. Existing codes such as the International Code of Zoological Nomenclature (ICZN) are character-based and rank-based and hardly allow conclusions on phylogenetic relationships. The 
PhyloCode establishes rules for applying names to clades and deliberately excludes information on the relative rank of clades. The advocates of the PhyloCode highlight a said stability of names contrasting with the denied continuity of nomenclature of traditional codes. This statement neglects that "specifiers" of clades are no longer valid once a supposed synapomorphy is recognized to be actually a homoplasy. Checks of further properties of the PhyloCode did not reveal demonstrable advantages over existing codes like the ICZN. The latter has shortcomings concerning the consideration of recent findings of phylogenetic research. This is by far outweighed by the fact that the underlying principle of the ICZN proved to be applicable as a mandatory code for 100 years and in agreement with all the scientific progress during 250 years.

DNA Barcoding is a method for using a small section of DNA to characterize all extant species. The resolution of the mitochondrial COI sequence and its storage in a data bank has been proclaimed to allow the unambiguous characterization and identification of all organisms. Supposedly, this should be a more efficient method to recognize and register the taxonomic biodiversity on earth when compared to the mostly non-molecular methods of traditional taxonomists. It turns out, however, that the base sequence analysis of COI of actually all species is a utopian goal. An unambiguous identification seems hardly possible in all cases because of a certain variability in the base sequences. COI analyses form one part of the molecular techniques and methods used by taxonomists for several years in addition to traditional methods. Thus, any effort to enhance COI analyses results in an addition to the knowledge on taxonomic diversity. Taxonomists working in an integrative manner by combining traditional and molecular methods are well aware that only a combination of several molecular and traditional methods contributes to a progress in revealing taxonomic diversity. Among the main goals of DNA Barcoding is the automated identification of taxa. Currently, this does not seem to be generally possible with an unambiguous result and, therefore, the whole range of molecular and traditional techniques will continue to be required for the characterization and identification of species.

\section{Keywords}

clade, DNA Barcoding, DNA taxonomy, ICZN, PhyloCode, phylogenetics, molecular systematics

\section{Einleitung}

Seit kurzem bewegen die Begriffe PhyloCode und DNA Barcoding die Gemüter von Taxonomen und die unmittelbar auf der biologischen Systematik aufbauenden Wissenschaften wie z. B. die Biogeographie. Der PhyloCode soll internationale Regeln für die phylogenetische Nomenklatur darstellen, indem er Regeln für die Benennung von Monoyphyla und anderen Kladen (gemäss Definition von WëGele, 2000, S. 71) zur Verfügung stellt. Das DNA Barcoding, auch als „Strichcode des Lebens“ bezeichnet, steht für eine Genabschnitt-Methode zur Kennzeichnung von Arten.

Jeden Systematiker, aber auch die meisten Biogeographen betreffen diese beiden neuen Entwicklungen unmittelbar in der täglichen Arbeit. Wir sind daher darauf angewiesen, uns eine Meinung dazu zu bilden. Die wissenschaftliche Literatur war in Kürze voll von Beiträgen Pro und Kontra dieser Regeln und Techniken und manchmal war gar nicht mehr deutlich, ob die Autoren wirklich wussten, worum es sich bei den Originalvorschlägen wirklich handelt. Auch in der Fachpresse wurden beide Themen kontrovers und oft personenbezogen diskutiert. Voraussetzung für eine wissenschaftliche Diskussion ist die Kenntnis der Originalvorschläge und der bisher erfolgten Weiterentwicklungen.

Der hier vorliegende Aufsatz stellt zu diesem Zweck die Grundlagen der beiden heiss diskutierten Themen in möglichst verständlicher Form dar. Dabei betrachte ich dies hauptsächlich aus dem Blickwinkel eines Entomologen. Weiterhin werden die von Gegnern wie Befürwortern vorgebrachten Argumente gebündelt und gegenübergestellt. Zwar 
wurde versucht, die wichtigsten Pro- und Kontra-Argumente auszuwählen, doch ist eine gewisse Subjektivität bei der Auswahl aus der Fülle der auf dem Markt befindlichen Argumente nicht völlig zu vermeiden. Schliesslich wird auch die eigene Position deutlich gemacht und die Begründung dafür gegeben, inwieweit es sich bei dem PhyloCode und dem DNA Barcoding tatsächlich um heute und in Zukunft relevante Regeln und Techniken handelt. Der Beitrag ist demnach ein sehr komprimiertes Überblicksreferat und gleichzeitig ein Positionspapier des Autors.

\section{PhyloCode}

\subsection{Gibt es überhaupt einen Anlass, sich über neue Regeln Gedanken zu machen?}

Die bis heute verbindliche zoologische Nomenklatur basiert auf der binären Namensgebung, die zum ersten Mal konsequent 1758 in der 10. Auflage von Linnés „Systema Naturae" angewendet wurde. Weitere Regeln umfassen die Internationalen Regeln für die Botanische Nomenklatur und die Nomenklatur von Bakterien und Viren. Die heute von der Unterart bis zur Überfamilie offiziell geregelte zoologische Nomenklatur hat seit 250 Jahren folgende Ziele:

- Ermöglichung der wissenschaftlichen Kommunikation. Beispiel: Unabhängig von der lokalen Sprache ist für jeden Wissenschaftler, wo auch immer auf der Welt, Cicindela campestris Linné, 1758, dieselbe Tierart.

- Darstellung der tatsächlichen, in der Natur vorhandenen hierarchischen Verwandtschaftsverhältnisse. Beispiel: Die zur selben Gattung gerechneten Arten sind untereinander näher verwandt (ursprünglich: einander ähnlicher) als jede von ihnen mit Arten einer anderen Gattung.

Seit 1905 existieren offizielle internationale Regeln für die zoologische Nomenklatur (aktuelle vierte Auflage: International Commission on Zoological Nomeclature, 1999; deutsche Ausgabe: Kraus, 2000), die über die beiden genannten Punkte hinaus vor allem folgendes Ziel verfolgen:

- Stabilität der Nomenklatur: Selbstverpflichtung der Wissenschaftsgemeinschaft, Namen von Tieren nur innerhalb festgelegter und nachvollziehbarer Verfahrensweisen zu vergeben (z. B. Prinzip des Holotypus, bi- bzw. trinäre Nomenklatur, Prioritätsprinzip zurück bis Linné, 1758).

Nun ergaben sich in 250 Jahren der Forschung zur zoologischen Systematik teils weitreichende neue Erkenntnisse. Diese umfassen vor allem die Notwendigkeit der Abkehr vom typologischen Konzept zu einer genealogischen Ordnung und damit von der Gleichsetzung von Ähnlichkeit mit Verwandtschaft zu einer evolutionären Sichtweise. Die aktuellen Regeln weisen einige Unzulänglichkeiten auf, von denen wenige hier kurz anhand der wünschenswerten Ziele behandelt werden sollen.

Die Namensgebung soll die genealogische Ordnung abbilden:

Es gibt um ein Vielfaches mehr Ränge als Namenskategorien zur Verfügung stehen, da nur 5 Kategorien der Familiengruppe, 2 der Gattungsgruppe und 2 der Artengruppe nomenklatorisch geregelt werden. Zwischen diesen offiziellen Kategorien gibt es abhängig von der Artenzahl weitere Kategorien. Auch unterhalb der Spezies bzw. Subspezies 
gibt es weitere Kategorien, wie Population und Dem. Weiterhin bilden die Namen der Kategorien die tatsächliche phylogenetische Verwandtschaft nur unvollständig ab, da innerhalb eines Rangs die Verwandtschaftsverhältnisse nicht erkennbar sind (Fig. 1, 2). $\mathrm{Ob}$ in einem Drei-Taxa-System die Verwandtschaftsverhältnisse $(A+B)+C$ oder $A+(B+C)$ lauten, kann nicht durch die Namensgebung deutlich gemacht werden. Gibt es also mehr als zwei Taxa innerhalb der selben Kategorie, so kann aus der Nomenklatur nicht auf deren Verwandtschaftsverhältnisse geschlossen werden. Drittens ist die Kategorisierung (d. h. das Ranking) bei den verschiedenen Taxa unterschiedlich und beruht trotz mancher Versuche, hierfür Kriterien zu erstellen (wie Anzahl der damit zusammengefassten Arten, Ähnlichkeit, Zeitpunkt der Aufspaltung), lediglich auf Konventionen.

Auch wird aus guten Gründen bei konsequenter Anwendung der phylogenetischen Systematik auf die Bezeichnung der Kategorie vor allem oberhalb der Familiengruppe (z. B. als Ordnung oder Klasse) verzichtet (z. B. Ax, 1984, 1999). Konsequenterweise müssten alle Monophyla von den Artengruppen bis zu den Organismenreichen mit einem eigenen Namen belegt werden. Die bekannte genealogische Ordnung wird heute meist als Kladogramm oder in Form hierarchisch angeordneter Namenslisten dargestellt.

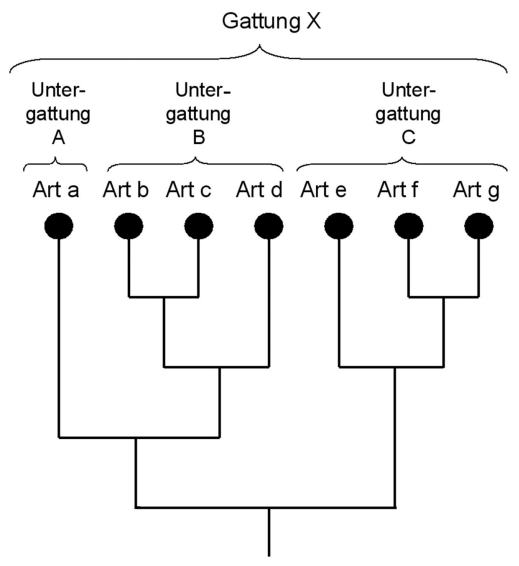

Gattung $X$ Untergattung A

Untergattung B

Art b

Art c

Art d

Untergattung $\mathrm{C}$

Art e

Art $\mathbf{f}$

Art $g$
Fig. 1: Die phylogenetische Verwandtschaft der Arten a bis g, der monophyletischen Untergattungen A bis C und der monophyletischen Gattung X, dargestellt als Kladogramm.

Fig. 2: Die Arten und höheren Ränge aus Fig. 1 als hierarchisch angeordnete Aufzählung der Namen. Die phylogenetischen Beziehungen der drei Untergattungen sowie der je drei Arten der Untergattungen B und C sind hieraus nicht ersichtlich. Falls es sich um eine gut bearbeitete Gattung handelt, kann allerdings zumindest unterstellt werden, dass neben der monobasischen Untergattung A auch die Untergattungen B und C Monophyla repräsentieren.

Fazit: Die Namensgebung auf der Basis der aktuellen Regeln bildet die genealogische Ordnung nur unvollständig ab. 
Die Namensgebung soll Arten klar gegeneinander abgrenzen:

Es gibt das nach wie vor ungelöste Problem des Artkonzepts und dessen praktische Umsetzung in eine Definition der Spezies, obwohl wir alle mit diesem Begriff wie selbstverständlich umgehen. Von den beiden heute am weitesten verbreiteten Konzepten ist das biologische Artkonzept nicht auf Agamospezies (uniparentale bzw. klonale Organismen) anwendbar und somit nicht universell gültig. Das phylogenetische Artkonzept ist zwar das derzeit wohl am besten abgesicherte Modell, aber es ermöglicht noch schwerer als die biologische Artdefinition eine praktisch umsetzbare Arterkennung. Unabhängig von diesen Artkonzepten stellt sich die Frage der Abgrenzung solcher taxonomischer Einheiten. Sollten nicht die „evolutionary significant units“ (ESU) mit Namen belegt werden und wie erkenne ich diese in der Praxis? Möchten wir durch die Namensgebung ein „species-sorting“ oder ein „gene-sorting“ darstellen? (vgl. Fig. 3). Die Abspaltung genetischer Linien kann der Populationstrennung vorausgehen und genetische Linien unterschiedlichen Beginns und Endes können sich in der selben oder in verschiedenen Populationen wiederfinden (Avise 2000). Arten werden durch den Holotypus definiert. Das Holotypus-Exemplar kann auch einem „Gen-Ast“ entstammen, der im Lauf der anagenetischen Entwicklung ausstirbt oder einer genetischen Linie, die nur in der Schwesterpopulation persistiert.

Fazit: Das auf der Artkategorie aufbauende Holotypus-Prinzip für die gesamte Nomenklatur basiert, um es überspitzt zu formulieren, auf einer nicht eindeutig definierten Gruppe von Individuen oder Populationen (nämlich der Spezies) und steht als Einzelindividuum (Paratypen bleiben hier unberücksichtigt) eher für das gene-sorting als das species-sorting in der Natur.

Fig. 3: „Species-sorting“ und „Gene-sorting“ können unterschiedlich verlaufen, Gen- und Artenstammbaum müssen nicht gleichförmig sein. Dennoch funktioniert meistens in der Praxis die Rekonstruktion der Phylogenese (vgl. Wägele, 2000) (nach Avise, 2000).

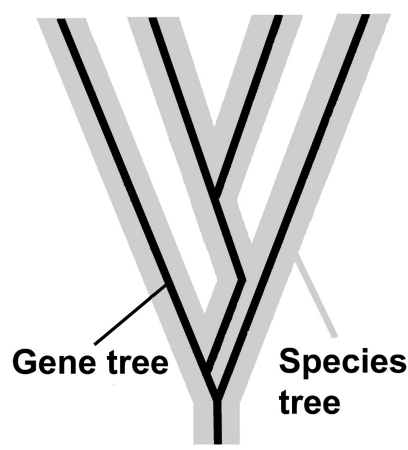

Fazit: Es ist durchaus nicht so, dass die existierenden nomenklatorischen Regeln in jeder Hinsicht ideal wären. War dies zu Linnés Zeiten noch anders, so kann doch heute aufgrund der weit grösseren Zahl bekannter Taxa und der Erkenntnisfortschritte bezüglich Evolution, insbesondere der Phylogenie, die genealogische Ordnung sowohl im Bereich der Artkategorie als auch auf allen übrigen hierarchischen Ebenen nur eingeschränkt dargestellt werden. Auch wenn Namen aufgrund von Revisionen geändert werden müssen, und die Kategorien, ohne dass dies wissenschaftlich begründbar wäre, je nach Ansicht des Autors ändern, so bleibt dies doch nachvollziehbar und belegt die Stabilität der Nomenklatur (wenn auch nicht die Permanenz gültiger Namen oder Kategorien). Die seit 1905 in Regeln gefasste, aber im Prinzip seit 250 Jahren 
existierende zoologische Nomenklatur stellt eine ausreichende Basis für eine Darstellung der Ordnung der organismischen Vielfalt und eine funktionierende wissenschaftliche Kommunikation darüber dar. Es ist zu fragen, ob auch heute noch der ursprüngliche Anspruch, die hierarchische bzw. heute genealogische Ordnung der Natur vollständig darzustellen, aufrecht erhalten werden muss. Die evolutionäre Systematik musste auch der phylogenetischen Systematik weichen, weil sie ihrem Anspruch, gleichzeitig die genealogische Ordnung wie auch Entwicklungsstufen darzustellen, nach objektiven Kriterien nicht gerecht werden konnte. Stabilität der Nomenklatur und wissenschaftliche Kommunikation über die organismische Vielfalt sind mit den existierenden Regeln gewährleistet. Darüber hinaus ermöglichen sie auch heute noch trotz stark veränderter Erkenntnislage zentrale, wenn auch unvollständige, Informationen über die Verwandtschaftsverhältnisse.

\subsection{Ziel und Struktur des PhyloCode}

Abgesehen von einigen Vorgängerpublikationen wurde die Idee eines PhyloCode durch De Queiroz \& Gauthier (1990) eingeführt. Die heutigen Hauptinitiatoren sind P. D. Cantino (Plant Biology, Ohio University, Athens) und K. de Queiroz (Vertebrate Zoology, National Museum of Natural History, Smithsonian Institution, Washington D.C.). Unterstützer finden sich vor allem unter den Biologen und Paläontologen der Yale University. Die Diskussion über den PhyloCode wird besonders im Internet geführt, aber auch in renommierten Zeitschriften wie Science oder Systematic Biology. Es gab mehrere Workshops, auf denen die Idee des PhyloCode diskutiert wurde und im Juli 2004 fand in Paris das 1st International Phylogenetic Nomenclature Meeting mit 70 Teilnehmern statt und dort wurde die International Society for Phylogenetic Nomenclature gegründet.

Das Ziel des PhyloCode ist die Einführung einer „Phylogenetischen Nomenklatur“. Diese beinhaltet

- die Etablierung eines universell für alle Organismen gültigen Systems (Bakterien, Pflanzen, Tiere [heute: Monera, Protista, Plantae, Fungi, Animalia]), einschliesslich der Viren.

- die Benennung der Organismenvielfalt auf der Basis der phylogenetischen Beziehungen („clade based“) anstatt von Merkmalen und deren hierarchischer Zusammenfassung („rank based“).

Einige der offensichtlichen Probleme mit diesen neuen Regeln werden dadurch umgangen, dass der PhyloCode zunächst parallel zu den bestehenden Regelwerken und nur oberhalb des Artniveaus etabliert werden soll. Der PhyloCode existiert derzeit (September 2005) als Internet-Publikation (CAntino \& De Queiroz, 2004).

Als Hauptanlass für die Einführung eines neuen Regelwerks werden die folgenden praktischen und theoretischen Gründe genannt:

- Die bisherigen Regeln fussen auf hierarchisch aufgebauten konkreten Rängen bzw. Kategorien, d. h. sie sind „rank-based“. Daher sind sie per se nicht dafür geeignet, die Benennung der natürlichen Einheiten, nämlich Arten und Monophyla, so zu regeln, dass Änderungen über die Zeit vermieden werden. Rangänderungen im traditonellen Regelwerk bedeuten automatisch - auch bei unveränderter Stellung im phylogenetischen System - eine Namensänderung. 
- Nur Monophyla (= "clades" gemäß Definition des PhyloCode) und Arten sind natürliche Einheiten (Taxa). Das bisherige System basiert aber auf künstlichen typologischen Prinzipien.

Die Namensgebung wird in der Praxis folgendermassen geregelt:

- Ein Monophylum bzw. ein Kladus = ein Wort (möglichst bereits existierender Name)

- Der Name muss mit einer phylogenetischen Definition (= „specifier“) versehen werden (Fig. 4, 5). Solche „specifiers“ können entweder konkrete Synapomorphien sein oder die Nennung der Einbeziehung bzw. der Einbeziehung und des Ausschlusses bestimmter Taxa.

- Eintrag des Namens und seiner phylogenetischen Definition in eine Datenbank.

Ein geänderter Kenntnisstand nach Revisionen kann zu einer Rangänderung, nicht jedoch zu einer Namensänderung eines einmal definierten Taxons führen.

Fig. 4: Drei Kladus-Typen mit ihren phylogenetischen Definitionen für die Errichtung von Kladus-Namen im Rahmen des PhyloCode (dazu kommen die nicht dargestellten „crown clades", d. h. wie im Beispiel der Knoten- oder Apomorphiebezogenen Definition, jedoch unter Einbezug nur der rezenten Taxa) (nach Angaben in Cantino \& De Queiroz, 2004).
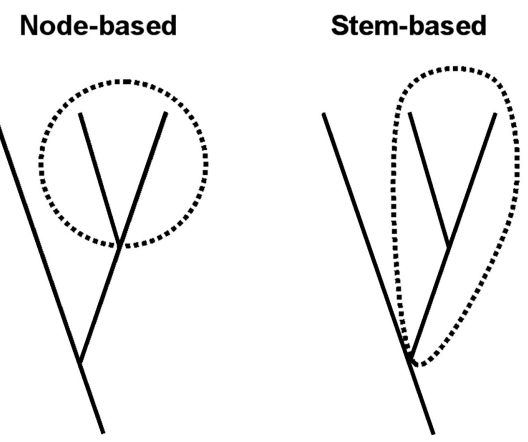

Apomorphy-based

Fig. 5: Beispiel für die phylogenetische Definition von Kladus-Namen durch das Spezifikationskriterium $(=$ „specifier") „Ein- bzw. Ausschluss bestimmter Taxa“ im Rahmen des PhyloCode: Definition von Lepidosauriformes: Monophylum (der Sauria), das Lacerta agilis Linnaeus, 1758, einschliesst, aber Youngina capensis Broom, 1914, nicht enthält (Cantino \& De QueIroz, 2004).

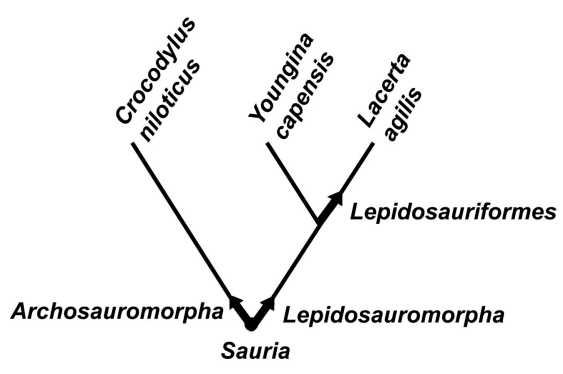

\section{3. „Evaluation“ des PhyloCode}

Dieses Kapitel kann aus Umfangsgründen keine echte Evaluation der Vor- und Nachteile des PhyloCode sein, weshalb der Begriff in der Kapitelüberschrift in Anführungszeichen gesetzt ist. Dennoch wird versucht, die wesentlichen Eigenschaften zentraler Eigenschaften des PhyloCode darzustellen und zu diskutieren.

Die folgende Aufzählung ist eine subjektiv ausgewählte Liste von Publikationen pro und kontra PhyloCode: Benton M. J., 2000; Nixon \& Carpenter, 2000; Löbl, 2001; Pleijel \& Rouse, 2003; Donoghue \& Gauthier, 2004; Kraus, 2004; Wenzel, J. W. u. a., 2004 . 
Im Gegensatz zum traditionellen Regelwerk können theoretisch alle Monophyla aller Ebenen mit Namen belegt werden. Diese Taxa-Namen können das beliebig Vielfache der Zahl von Artnamen erreichen, weshalb sie nur mit Hilfe einer elektronischen Datenbank zu handhaben sind.

Aus den unter dem PhyloCode erstellten Namen sind keine Ränge ablesbar und somit geben sie auch keine Informationen über die relative Stellung im Kladogramm, d. h. innerhalb der genealogischen Ordnung.

Neben der Benennung von Arten ist die ausschliessliche Benennung von Monophyla, also durch Synapomorphien belegte geschlossene Abstammungsgemeinschaften, seit langem auch das explizite Ziel der traditionellen zoologischen Nomenklatur. Hier stehen im offiziell geregelten Bereich nur relativ wenige Kategorien zur Verfügung, der PhyloCode verwendet dagegen überhaupt keine Ränge.

Vermeintlich gut belegte Synapomorphien können sich bei Revisionen wie bisher auch als Konvergenzen oder andere Homoplasien herausstellen. Der durch einen solchen „specifier“ begründete Name wird damit ungültig. Namensänderungen werden damit auch unter dem PhyloCode notwendig.

Die zur Kennzeichnung der Kladen verwendeten Synapomorphien sind letztlich auch Eigenschaften von Merkmalen und die als „specifiers“ verwendeten Arten sind auch über Merkmale definiert. Der PhyloCode ist somit auch merkmalsbasiert und nicht ausschliesslich über die phylogenetischen Beziehungen, also „clade-based“ definiert.

Die Idee, alle real in der Natur vorkommenden Taxa (Arten und Abstammungsgemeinschaften) über alle Organismengruppen hinweg mit einem einheitlichen, stabilen System zu benennen, ist sicher sinnvoll. Es gab auch früher schon solche Versuche, wie den BioCode (Greuter u. a., 1996), die jedoch keine Praxisreife und allgemeine Akzeptanz erreichten. Es gibt bisher keinen Hinweis darauf, dass der PhyloCode diesem Anspruch gerecht werden könnte. Der PhyloCode wird auch weiteren wünschenswerten Zielen nicht gerecht: Die Abbildung der Hierarchie in der genealogischen Ordnung fehlt (bewusst) völlig, während sie durch das aktuelle Regelwerk zumindest teilweise deutlich gemacht wird. Die mit dem Artstatus verbundenen Probleme führten im Phylocode zum derzeit völligen Ausklammern des Artniveaus, so dass nur die traditonellen Regeln hier Anwendungen anbieten. Im Gegensatz zu den traditionellen Regeln stellt der PhyloCode Anleitungen zur Benennung sämtlicher Kladen zur Verfügung: Aufgrund der mit dem ICZN benannten (mehr als zwei) Untergattungen ist deren phylogenetische Beziehung untereinander nicht ablesbar, während die unter dem PhyloCode erstellten Namen theoretisch alle Kladen eindeutig kennzeichnen. Allerdings nützt das nichts in der Praxis, da im Gegensatz zum traditionellen System deren Position innerhalb des Kladogramms überhaupt nicht angezeigt wird.

Fazit: Der PhyloCode bietet an keinem Punkt einen verifizierbaren Fortschritt gegenüber traditionellen Regelwerken wie dem ICZN, während das Prinzip der letzteren seit 250 Jahren und ein dezidiertes Regelwerk seit 100 Jahren erfolgreich angewendet wird. Die Wissenschaftsgemeinschaft muss weiter daran arbeiten, die bereits bekannten und die noch zu entdeckenden Erkenntnisse der Systematik optimal in die bestehenden Regelwerke einzubringen. Dabei muss aber jede Änderung bestehender Regeln einen nachweisbaren Vorteil bringen. Es scheint mir vielversprechend, zwischen der wissenschaftlichen Kommunikation über Taxa auf der Basis einer stabilen Nomenklatur mit lediglich Basisinformationen zur Phylogenie auf der einen Seite und detaillierten Informationen zur 
phylogenetischen Verwandtschaft auf der anderen Seite zu unterscheiden. Erstere Aufgabe kann in sehr guter und bewährter Weise durch die bestehenden Regelwerke gewährleistet werden, letztere kann wie bisher auch als begleitende pragmatische Darstellung in Form eines Kladogramms (mit beliebig vielen Ebenen von Detailinformationen) dargestellt werden. Damit möchte ich nicht die Idee eines universellen BioCode begraben (obwohl das bisherige Projekt aufgegeben wurde), sondern einen solchen auf das realistische Ziel der Erleichterung der wissenschaftlichen Kommunikation reduzieren, und umfangreiche phylogenetische Informationen gar nicht erst als Ziel eines solchen universellen Codes aufzunehmen.

Der PhyloCode ist in der aktuell verfügbaren Form wie auch vom Ansatz her nicht praktikabel. Auffallend und beunruhigend ist jedoch, dass manche Vertreter dieser Idee bewährte Systeme und Regelwerke negativ belegen (diese würden auf dem anachronistischen Aristotelischen Essentialismus basieren, deren Anhänger seien demnach „essentialists“, „typologists“ oder „Linnean taxonomists“), ohne eine abgesicherte Alternative zur Verfügung zu stellen - und vor allem bei Nicht-Taxonomen Fragen zur Biodiversität negativ beeinflussen können. Eine Umfrage unter den Autoren des Catalogue of Palaearctic Coleoptera ergab, dass $50 \%$ den PhyloCode als unnötig und $8 \%$ ihn als nützlich erachteten (LöBl \& LesCHen, 2005). Der ICZN wird auch weiterhin das verbindliche Regelwerk bleiben, doch reicht ein einfaches Ignorieren des PhyloCode nicht aus. Insbesondere die Schriftleiter wissenschaftlicher Zeitschriften sind hier gefordert, auf die Einhaltung des ICZN zu achten, um auch weiterhin eine wissenschaftliche Kommunikation zu ermöglichen.

\section{DNA Barcoding}

\subsection{Grundlagen}

Mutationen manifestieren sich auf molekulargenetischer Ebene durch den Verlust, den Zugewinn oder den Austausch einer der vier Basen der DNA. Beim genetischen Code ist vor allem die dritte Codon-Position wenig durch die Selektion betroffen (d. h. u. a., dass die Aminosäure durch Austausch der dritten Base nicht geändert wird), d. h. Basenänderungen können sich (fast) nur hier etablieren (vgl. Wobble Position, Fig. 10). In nicht codierenden DNA-Abschnitten können sich wegen der geringeren Selektion im Lauf der Evolution die Basensequenzen stärker verändern als in codierenden Abschnitten. Im Vergleich der DNA unterschiedlicher Organismen durch Alinierungsverfahren lassen sich Ähnlichkeiten in den Basensequenzen ermitteln (Fig. 6). Diese können bei ent-

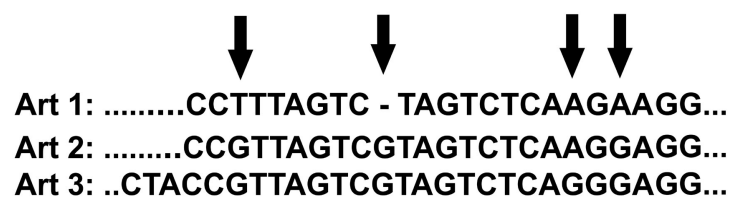

Fig. 6: Durch Vergleich der DNA unterschiedlicher Organismen lassen sich Ähnlichkeiten in den Basensequenzen bei entsprechender Analyse als „Verwandtschaftsgrad“ interpretieren. Im Rahmen von Alinierungsverfahren wird die Abfolge der Basen der DNA einzelner Individuen miteinander verglichen (hier abgebildetes Beispiel: nicht codierender Abschnitt). Die Pfeile weisen auf Substitutionen bzw. den Ausfall einzelner Basen (nach Mossakowski, 1997). 
sprechender Analyse als Verwandtschaftsgrad interpretiert werden. Je nach Fragestellung (Aufklärung der phylogenetischen Beziehungen auf Populationsebene, Artebene oder zwischen umfassenderen, d. h. auch älteren Abstammungsgemeinschaften) werden DNA-Abschnitte unterschiedlicher Herkunft (Mitochondrien oder Kern) und unterschiedliche Gene bzw. Basensequenzen analysiert. Längere Basensequenzen und die kombinierte Untersuchung mehrerer Gene erhöhen die Qualität der Aussage. Die ringförmig angeordnete mitochondriale DNA wird rein maternal vererbt und hat im Vergleich zu Kern-DNA eine etwa zehnfach höhere Mutationsrate und zeigt eine stärkere genetische Drift (ca $5 \%$ Substitutionsrate pro $10^{6}$ Jahre) (Fig. 7). Daher eignen sich mitochondriale Gene zumindest theoretisch besonders gut für eine höhere zeitliche Auflösung, d. h. zur Feststellung jüngerer phylogenetischer Aufspaltungsereignisse wie etwa das Artoder Gattungsniveau. Der Cytochrom $c$ - Oxidase I (COI) codierende Abschnitt der mitochondrialen DNA gehört mit mehr als 1500 Basenpaaren (bp) zu den längeren Basensequenzen. Für das DNA Barcoding wird davon meist ein Abschnitt von 648 bp verwendet. Dieses COI Gen diente bereits in zahlreichen Arbeiten zur Aufklärung der phylogenetischen Beziehungen vom Art- bis zum Familienniveau (Fig. 8). Diese und vergleichbare Nutzung der DNA in der Systematik und Biogeographie ist heute weit verbreitet und unumstritten.

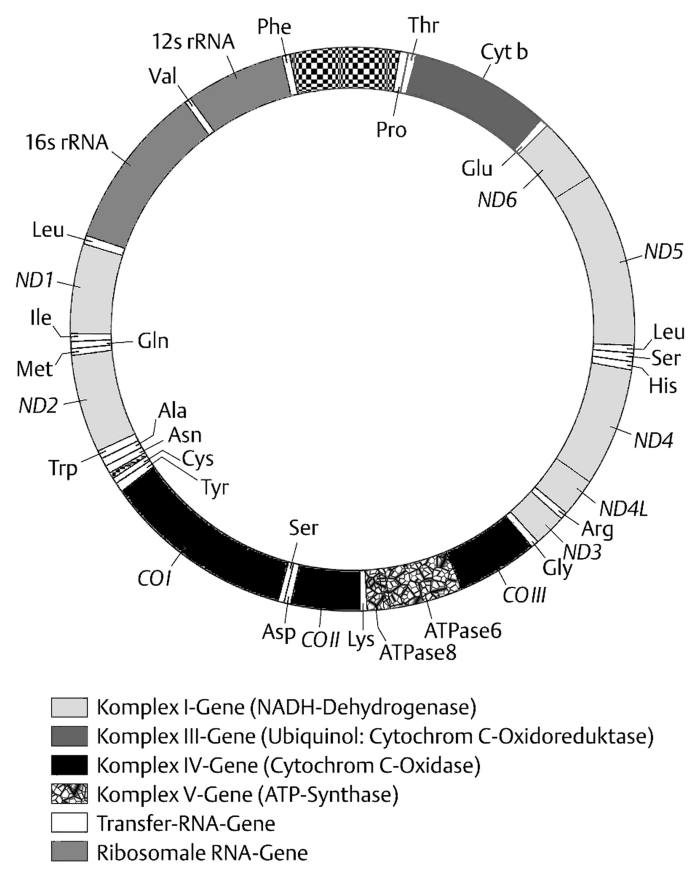

Fig. 7: Das ringförmige Mitochondriengenom (hier: des Menschen) mit Lage des Gens für die Untereinheit I der Cytochrom $c$ - Oxidase (COI) (JANNING \& KNUST , 2004, verändert).

\subsection{Was unterscheidet das DNA Barcoding von den etablierten Ansätzen?}

Die unter 3.1 genannten Grundlagen, Methoden und Techniken der molekularen Systematik sind die selben, die auch beim DNA Barcoding zum Ansatz kommen. Das DNA Barcoding unterscheidet sich von diesen Ansätzen nicht in der verwendeten Technik, sondern lediglich in folgender Zielsetzung: 
- COI-Sequenzen dienen primär der automatischen Identifizierung von Taxa.

- Dies erfordert eine Datenbank, in die möglichst rasch die COI-Sequenzen möglichst aller bekannten Tiere und Pflanzen eingegeben werden.

- Dies wiederum erfordert den intensiven Ausbau möglichst vieler molekulargenetischer Laboratorien und deren Koordination.

Der Begriff „barcoding“ bezieht sich auf den Begriff „bar code“, also den elektronisch lesbaren Strichcode zur individuellen Kennzeichnung z. B. von Waren (Fig. 9). Bei die-

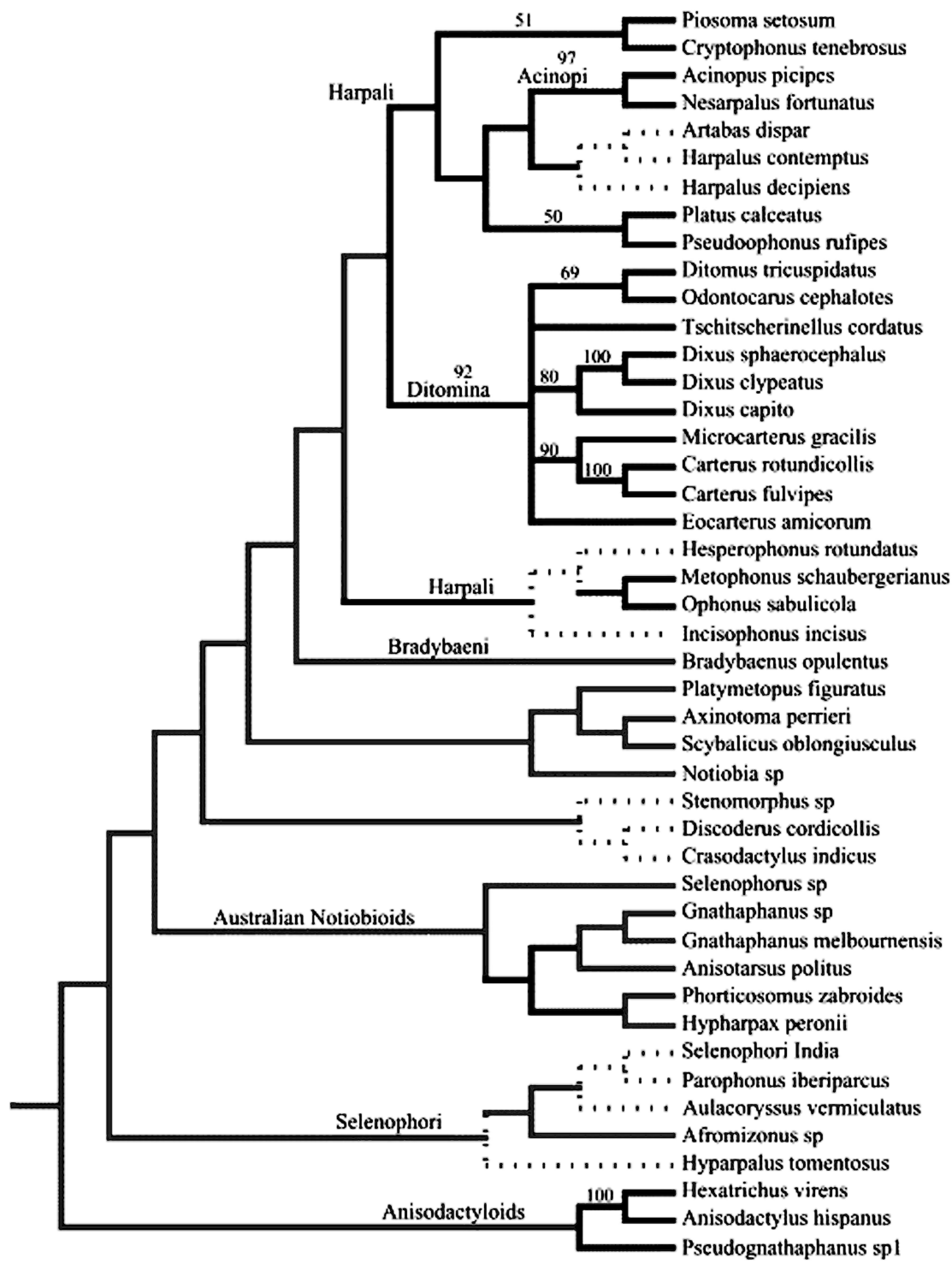

Fig. 8: Beispiel für die Verwendung des mitochondrialen Cytochrom $c$ - Oxidase I Gens (COI) zur Rekonstruktion der Phylogenese der Harpalini (Carabidae) (abgebildet ist ein Ausschnitt aus dem strikten Konsensusbaum einer ungewichteten Maximum Parsimony Analyse) (aus Martinez-Navarro u. a., 2005). 
sem Strichcode reichen 10 Zeichen auf 12 Positionen aus, um 1 Billion unterschiedlicher Produkte zu kennzeichnen. Trotz der bei den Organismen vorhandenen Einschränkungen in der freien Variabilität (keine Base kann mit der gleichen Wahrscheinlichkeit durch eine der drei anderen ersetzt werden oder ausfallen) sind die 4 "Zeichen“ (= Basen) an jeder dritten Position entlang der ausgewählten 648 bp von COI theoretisch mehr als ausreichend, um alle Arten von Organismen zu identifizieren (Fig. 10).
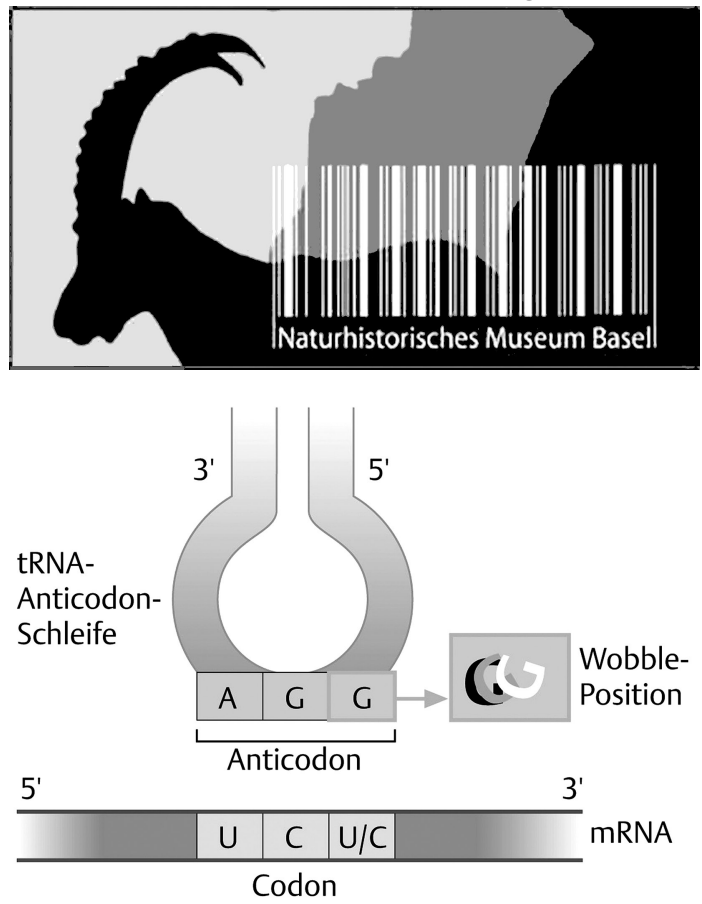

Fig. 9: Symbolische Darstellung des „Strichcodes des Lebens" (Ausschnitt des Posters zur 8. Jahrestagung der Gesellschaft für biologische Systematik vom 13. bis 16.9.2005 in Basel). Bei einem einfachen Produkt-Strichcode reichen 10 Zeichen auf 12 Positionen aus, um 1 Billion unterschiedliche Produkte zu kennzeichnen.

Fig. 10: Die „Wobble”-Base, d. h. die Base an der ersten Stelle (5'-Ende) des Anticodons in der tRNA kann verschiedene Basenpaarungen mit der dritten Position des Codons der mRNA eingehen. Daher ist die dritte Position eines Codons weniger stark der Selektion unterworfen und Mutationen können hier besonders häufig auftreten (aus JANNING \& KNUST, 2004, verändert).

Der Begriff „Strichcode des Lebens“ (Barcode of Life) steht für den Anspruch der Förderer des DNA Barcoding, Organismen in Zukunft anhand eines kurzen Abschnitts mitochondrialer DNA mit Hilfe automatisierter Verfahren eindeutig identifizieren und kennzeichnen zu können. Eine in Grossbritannien 2003 patentierte „DNA barcoding technique“, mit deren Hilfe gentechnisch veränderte Organismen z. B. in Nahrungsmitteln anhand einer zusätzlichen gentechnischen Veränderung erkannt werden können, hat mit dem hier besprochenen „Strichcode des Lebens“ nichts zu tun.

Die Initiative für den Barcode of Life stammt von Paul D.N. Hebert, Zoology, University of Guelph, Kanada, und wurde 2003 zum ersten Mal publiziert (HeBert u. a., 2003). Die Unterstützergemeinde ist gross und dazu zählt z. B. auch die Deutsche Zoologische Gesellschaft. Die Diskussion darüber wird kontrovers, intensiv und umfangreich geführt sowohl im Internet als auch in renommierten wissenschaftlichen Zeitschriften wie Nature oder Cladistics. Mehrere Kongresse, Symposien oder Workshops widmeten sich diesem Thema, darunter solche am Naturhistorischen Museum London und der Zoologischen Staatssammlung München. Die Unterstützer dieser Initiative haben sich zusammengeschlossen zu einem Consortium for the Barcode of Life, National Museum of Natural History, Smithsonian Institution, Washington D.C., eine Website existiert unter www.barcodinglife.com. 


\subsection{Argumente und Gegenargumente für die Unterstützung des DNA Barcoding}

Die folgende Aufzählung von Publikationen ist eine subjektive Auswahl zur Verdeutlichung der kontroversen Diskussion dieser Thematik, wobei die Positionen von „DNA barcoding is no substitute for taxonomy“ (ЕвACH \& Holdege, 2005) über „DNA barcoding does not compete with taxonomy“ (Gregory, 2005) bis „DNA barcoding a useful tool for taxonomists" (Schindel \& Miller, 2005) reichen: TAutz u. a., 2002; Stoeckle, 2003; Moritz \& Cicero, 2004; Will \& Rubinoff, 2004; Wheeler, 2005; Jolivet, 2006.

Im folgenden werden einige mir wichtig erscheinende Argumente für das DNA-Barcoding aufgelistet und mit Gegenargumenten konfrontiert.

Argument a: Es gibt nicht genügend (traditionelle) Taxonomen, um die gesamte Biodiversität der Erde zu erfassen, bevor vieles wieder verschwunden ist. Die Erkennung und Beschreibung von Arten ist sehr kompliziert und zeitaufwendig. Auch in den letzten 250 Jahren gelang es nur, einen Bruchteil der lebenden Biodiversität darzustellen. Die automatisierte COI-Analyse stellt eine ungleich effektivere Methode zur Erfassung der biologischen Diversität und zur Bestimmung undeterminierten biologischen Materials dar.

Gegenargument a': Auch die COI-Sequenz (oder vergleichbare Technik) liefert selbst bei der Utopie einer vollständigen Datenbank aller bekannten Arten nur teilweise eine wirklich sichere Zuordnung zu einer Art. Im überwiegenden Teil der Fälle mit deutlich über 90 \% aber weniger als $100 \%$ Übereinstimmung in der Sequenz können nur (traditionelle) Spezialisten des betreffenden Taxons eine gesicherte Identifizierung vornehmen. Es gibt keine festen Grenzen bezüglich Unterschieden der COI-Sequenzen. Speziell für Pflanzen ist dieser DNA-Abschnitt nicht (gut) geeignet.

Argument b: DNA Barcoding kann als Ergänzung zu anderen Daten dienen, um z. B. Artgrenzen besser definieren zu können.

Gegenargument b': Dies ist zweifellos korrekt, doch ist dies bereits mit den derzeit bestehenden Datenbanken (z. B. bei EMBO, NCBI) oder durch gezielte Einzelprojekte möglich. Ein grosses Projekt „Barcode of Life“ ist hierfür nicht erforderlich.

Argument c: DNA Barcoding kann als Sortierwerkzeug benutzt werden, um unbekannte „Morphospezies“ (z. B. Nematoden aus einer Bodenprobe) bereits beschriebenen Arten zuzuordnen und neue Arten zu erkennen und mit Hilfe traditioneller Taxonomen zu beschreiben.

Gegenargument c': Auch hier funktioniert die positive Identifizierung nur bei vollständiger Datenbank und 100 \%iger Übereinstimmung. Der Hinweis auf möglicherweise neue, noch unbeschriebene Arten ist ein wichtiger Beitrag zur Erweiterung der Kenntnisse über die Biodiversität.

Argument d: DNA Barcoding trägt dazu bei, unbekannte Proben bekannten Arten zuzuordnen, z. B. wenn nur Körperteile oder Gewebereste vorliegen, beim Auftreten von Sexualdimorphismus oder individuellen Fehlbildungen oder der Zuordnung unbeschriebener Larven zu nur als Adulte bekannten Taxa.

Gegenargument d': Auch dies ist korrekt, doch funktioniert das bereits ohne DNA Barcoding mit den heute verfügbaren Handwerkszeugen (Fig. 11). 


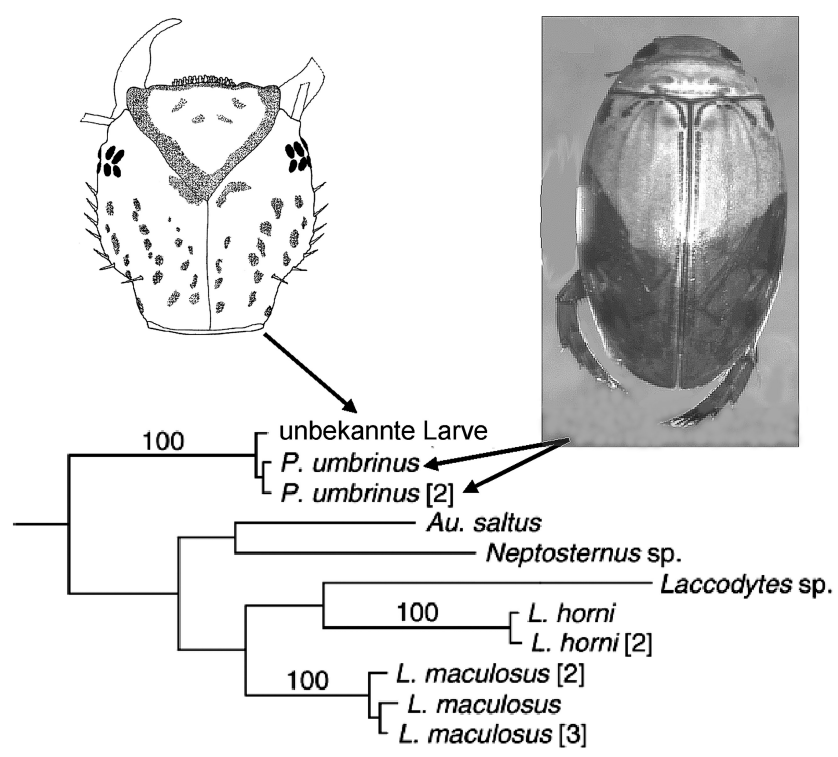

Fig. 11: Beispiel für die Verwendung des mitochondrialen Cytochrom $c$ - Oxidase I Gens (COI) zur Identifizierung unbekannter Larven, d. h. ihrer Zuordnung zu beschriebenen Imagines, hier der Art Philodytes umbrinus (Mотschulsky, 1855) Dytiscidae: Laccophilinae) (Ausschnitt eines Kladogramms auf der Basis einer Parsimony-Analyse der COI Sequenzen) (aus Miller u. a. 2005).

Argument e: DNA Barcoding ersetzt weitgehend die traditionelle Taxonomie, da nur mit dieser Methode innerhalb überschaubarer Zeit die gesamte Biodiversität erfassbar ist.

Gegenargument e': Diese Position wird heute nur noch von ganz wenigen Unterstützern des Barcoding vertreten. Es gibt zahlreiche Argumente dafür, dass die Biodiversität 1 . nicht ausschliesslich anhand eines Genabschnitts ausreichend beschrieben werden kann (s. o.) und 2. die Vielzahl der molekulargenetisch zu bearbeitenden Taxa überhaupt nur von integrativ arbeitenden Taxonomen erkannt und zur Verfügung gestellt werden kann.

Argument f: Die traditionelle Taxonomie erhält sowieso kaum öffentliche oder private Forschungsgelder, dagegen könnten durch ein umfangreiches DNA Barcoding Programm neue Finanzierungsmöglichkeiten erschlossen werden, die letztlich auch dem Ziel der traditionellen Taxonomie dienen.

Gegenargument f': DNA Barcoding behindert die integrativ-taxonomische Arbeit zur Erfassung der Biodiversität, weil diese Methode grosse finanzielle und personelle Ressourcen bindet, dabei aber nur einen kleinen Teil zum Ziel, nämlich der Beschreibung der Vielfalt des Lebens, beiträgt. Staatliche Forschungsgelder wie Stiftungsgelder können nur einmal ausgegeben werden und gehen, sofern sie für das Barcoding verwendet werden, theoretisch der übrigen molekularen wie auch der traditionellen Taxonomie verloren.

Fazit: Die allerjüngsten Stellungnahmen innerhalb dieser sehr rasanten Entwicklung deuten darauf hin, dass die ursprünglichen Ziele höchstens noch von ganz wenigen Fachvertretern verfolgt werden. Viele Wissenschaftler sind heute der Ansicht, dass die COI-Analyse lediglich als eine von mehreren Techniken der molekularen Systematik wie 
der Analyse weiterer mtDNA oder Basensequenzen der Kern-DNA, einschliesslich der Verwendung von Mikrosatelliten, anzusehen ist. Die phylogenetische Verwandtschaft und damit die genealogische Ordnung ist oft nur durch eine Kombination mehrerer solcher Methoden und Techniken wahrscheinlich zu machen. Anscheinend hat aber die für Forschungsförderer zunächst sehr attraktive Idee eines "Strichcodes des Lebens“ auch Investitionen in molekularbiologische Laboratorien und in die Ausbildung von Wissenschaftlern ermöglicht, die heute auch der gesamten molekularen Systematik zu gute kommt.

Der Begriff der DNA-Taxonomie wird neuerdings bezüglich Ziel und Technik mit dem DNA Barcoding mehr oder weniger gleichgesetzt (vgl. TAUTZ u. a., 2002, sowie Stellungnahme der DZG u. a. 2005). Aus diesem Grund wurde im vorliegenden Beitrag bewusst auf diesen Begriff verzichtet, um eine Verwechslung mit den verschiedenen Strategien, Methoden und Techniken der molekularen Systematik zu vermeiden. Letztere ist wesentlich mehr als in der o.g. Stellungnahme unter DNA-Taxonomie beschrieben wird. (Ich verwende hier in Übereinstimmung mit Ax, 1984, die Begriffe Taxonomie und Systematik als Synonyme, da die Überlappung zu breit und in der Praxis keine klare Trennung möglich ist, vgl. aber z. B. WëGELE, 2000). Warum nun speziell die DNA-Taxonomie dieser Definition gefördert werden soll anstatt der umfassenderen molekularen Systematik, die doch bereits schon den Beweis ihrer Effektivität durch wichtige neue Forschungsergebnisse geliefert hat, bleibt für mich unklar. Eine Umfrage unter den Autoren des Catalogue of Palaearctic Coleoptera ergab, dass $78 \%$ molekulare Methoden in der Systematik als potentiell nützlich erachten, doch nur $23 \%$ halten das DNA Barcoding für nützlich (LöBL \& LEsCHEN, 2005).

Die hinter dem DNA Barcoding stehenden molekulargenetischen Methoden sind ein weiteres Hilfsmittel moderner Systematik und Taxonomie. Die umfassende Entschlüsselung der für COI verantwortlichen Basensequenzen möglichst vieler Organismen ist auch für die allgemeine Systematik-Forschung von grossem Vorteil. Es ist sicher nichts gegen die Einrichtung einer COI-Datenbank einzuwenden, sofern diese mit bestehenden Datenbanken verknüpft wird, doch erscheint deren Etablierung als COI-Datenbank aller Organismen unrealistisch. Die Möglichkeit der Identifizierung aller Arten allein mit Hilfe dieses Werkzeugs, selbst wenn andere DNA-Abschnitte mit einbezogen werden, wird nicht ausreichend durch Fakten unterstützt. Die ursprünglich proklamierte Ablösung der traditionellen Taxonomie durch das DNA Barcoding wird ernsthaft heute kaum mehr in Erwägung gezogen. Die "traditionelle“ Taxonomie hatte sich bereits vor der Proklamierung des DNA Barcoding zu einer „integrativen“ Taxonomie entwickelt, in der molekulargenetische Techniken ebenso selbstverständlich zur Anwendung kommen wie physiologische oder morphologische Kriterien.

\section{Danksagung}

Ich danke meinen Kollegen Roland Molenda und Alexander Szallies für die anregenden Diskussionen über das DNA Barcoding sowie Dirk Ahrens für die Literaturhinweise.

\section{Literaturverzeichnis}

Avise, J. C. 2000: Phylogeography. - Cambridge MA, London: Harvard University Press, VIII + 447 S. Ax, P. 1984: Das Phylogenetische System. Systematisierung der lebenden Natur aufgrund ihrer Phylogenese.

- Gustav Fischer Verlag, Stuttgart, New York: 349 S. 
Ax, P. 1999: Das System der Metazoa II. - Stuttgart, Jena: Gustav Fischer Verlag, 383 S.

Benton, M. J. 2000: Stems, nodes, crown clades, and rank-free lists: is Linnnaeus dead? - Biological Reviews 75: 633-648.

Cantino, P. D. \& De Queiroz, K. 2004: PhyloCode: A Phylogenetic Code of Biological Nomenclature. Version 2b. - www.ohiou.edu/phylocode/ (accessed August 2005).

De Queiroz, K. \& Gauthier, J. 1990: Phylogeny as a central principle in taxonomy: phylogenetic definitions of taxon names. - Systematic Zoology, Washington 39: 307-322.

Donoghue, M. J. \& Gauthier, J. A. 2004: Implementing the PhyloCode. - Trends in Ecology and Evolution 19 (6): 281-282.

Eвach, M. C. \& Holdrege, C. 2005: DNA barcoding is no substitute for taxonomy. - Nature 434: 697.

Gregory, T. R. 2005: DNA barcoding does not compete with taxonomy. - Nature 434: 1067.

Greuter, W.; Hawksworth, D. L.; McNeill, J.; Mayo, M. A.; Minelli, A.; Sneath, P. H. A.; Tindall, B. J.; Trehane, P. \& Tubbs, P. (eds.) 1996: Draft BioCode: The prospective international rules for the scientific names of organisms. - Taxon 45: 349-372.

Hebert, P. D. N.; Cywinska, A.; Ball, S. L. \& De Waard, J. R. 2003: Biological identifications through DNA barcodes. - Proceedings of the Royal Society of London, Series B, Biological sciences, London 270: 313-321.

International Commission on Zoological Nomeclature 1999: International Code of Zoological Nomeclature. Fourth Edition. London: The International Trust for Zoological Nomenclature c/o The Natural History Museum, XXIX + 306 S.

Janning, W. \& Knust, E. 2004: Genetik. - Georg Thieme Verlag, Stuttgart, New York: XIV+ 472 S.

Jolivet, P. 2006: Nomenclature and Nomenclatura. Taxonomy, Taxinomy or Systematics. - Nouvelle Revue d'Entomologie. Nouvelle Serié, Paris 22 (4): 355-360.

Kraus, O. 2000: Internationale Regeln für die zoologische Nomenklatur. Vierte Auflage. Offizieller deutscher Text. - Abhandlungen des Naturwissenschaftlichen Vereins Hamburg (NF) 34 - Keltern: Goecke \& Evers, $232 \mathrm{~S}$.

Kraus, O. 2004: Phylogeny, classification, and nomenclature: a reply to F. Pleijel and G. W. Rouse. - Journal of Zoological Systematics and Evolutionary Research, Blackwell Science, Berlin 42: 159-161.

LöвL, I. 2001: Les Nomenclatures "linnéenne" et "phylogénétique", et d’autres Problèmes artificiels. Bulletin de la Société française de Systématique 26: 16-21.

Löbl, I. \& Leschen, R. A. B. 2005: Demography of Coleopterists and their Thoughts on DNA Barcoding and the PhyloCode, with Commentary. - The Coleopterists Bulletin 59 (3): 284-292.

Martinez-Navarro, E. M.; Galian, J. \& Serrano, J. 2005: Phylogeny and molecular evolution of the tribe Harpalini (Coleoptera, Carabidae) inferred from mitochondrial cytochrome-oxidase I. - Molecular Phylogenetics and Evolution 35: 127-146.

Miller, K. B.; Alarie, Y.; Wolfe, G. W. \& Whiting, M. F. 2005: Association of insect life stages using DNA sequences: the larvae of Philodytes umbrinus (Mотschulsky) (Coleoptera: Dytiscidae). - Systematic Entomology, Oxford 30: 499-509.

Moritz, C. \& Cicero, C. 2004: DNA Barcoding: Promise and Pitfalls. - PLoS Biology 2 (10): 1529-1531.

Mossakowski, D. 1997: Klassische oder molekulare Methoden? Ihre Rolle in der modernen Evolutionsbiologie. - Veröffentlichungen aus dem Übersee-Museum Bremen, Naturwissenschaften, 13: 31-46.

Nixon, K. C. \& Carpenter, J. M. 2000: On the Other "Phyogenetic Systematics". - Cladistics, London 16: 298-318.

Platnick, N. I. 2001: From Cladograms to Classifications: The Road to DePhylocode. www.systass.org/ events_archive/agm-address-dec2001.html (accessed August 2005)

Pleijel, F. \& Rouse, G. W. 2003: Ceci n'est pas une pipe: names, clades, and phylogenetic nomenclature. - Journal of Zoological Systematics and Evolutionary Research, Blackwell Science, Berlin 41: 162-174.

Schindel, D. E. \& Miller, S. E. 2005: DNA barcoding a useful tool for taxonomists. - Nature 435: 17. 
Stellungnahme der Deutschen Zoologischen Gesellschaft, der Gesellschaft für Biologische Systematik und der Deutschen Botanischen Gesellschaft zur Förderung der DNA-Taxonomie (2005): GfBS News 15: 22-23.

Stoeckle, M. 2003: Taxonomy, DNA, and the Bar Code of Life. - BioScience 53 (9): 2-3.

Tautz, D.; Arctander, P.; Minelli, A.; Thomas, R. H. \& Vogler, A. P. 2002: A plea for DNA taxonomy. Trends in Ecology and Evolution 18 (2): 70-74.

WÄGele, J.-W. 2000: Grundlagen der Phylogenetischen Systematik. - Verlag Dr. Friedrich Pfeil, München: $315 \mathrm{~S}$.

Wenzel, J. W.; Nixon, K. C. \& Cuccodoro, G. 2004: Dites non au PhyloCode! - Bulletin de la Société française de Systématique 31: 19-23.

Wheeler, Q. D. 2005: Losing the plot: DNA “barcodes" and taxonomy. - Cladistics, London 21: 405-407.

Will, K. W. \& Rubinoff, D. 2004: Myth of the molecule: DNA barcodes for species cannot replace morphology for identification and classification. - Cladistics, London 20: 47-55.

\section{Anschrift des Verfassers:}

Prof. Dr. Peter Nagel

Institut für Natur-, Landschafts- und Umweltschutz /

Biogeographie, Department Umweltwissenschaften

Universität Basel

St. Johanns-Vorstadt 10

$\mathrm{CH}-4056$ Basel

Schweiz

peter.nagel@unibas.ch

www-nlu.unibas.ch

\section{Subject editor:}

L. ZerChe 